\title{
Stability of a Fermented Milk Enriched With Microbial CLA/CLNA
}

\author{
Ana L. Fontes ${ }^{1,2}$, Lígia L. Pimentel ${ }^{1}$, Luis M. Rodríguez-Alcalá1, Ana M. Gomes ${ }^{1}$ \\ ${ }^{1}$ Universidade Católica Portuguesa, CBQF - Centro de Biotecnologia e Química Fina - Laboratório Associado, Escola \\ Superior de Biotecnologia, Rua Diogo Botelho 1327, 4169-005 Porto, Portugal \\ ${ }^{2}$ Laboratório Associado para a Química Verde - LAQV-REQUIMTE, Departamento de Química, Universidade de Aveiro, \\ Campus Universitário de Santiago 3810-193 Aveiro, Portugal \\ afontes@ucp.pt; 1pimentel@ucp.pt; lalcala@ucp.pt; amgomes@ucp.pt
}

\section{Extended Abstract}

There is an increasing interest towards the development of innovative value-added food products with a potential to prevent or counteract disease conditions, many times impelled by unbalanced diets, such as cardiovascular diseases and cancer. Several promising food-derived lipids with potential bioactive properties have been identified over the last decade, and these include conjugated linoleic (CLA) and conjugated linolenic (CLNA) acids [1], [2]. Due to concentration and availability limitations in their natural sources (e.g. ruminants' milk and meat or vegetable oils) [3], [4], the in situ microbial production may reveal itself to be a good strategy to increment CLA/CLNA daily intake. Several probiotic strains have demonstrated the ability to produce CLA/CLNA isomers using linoleic (LA) and alpha-linolenic ( $\alpha$-LNA) acids as precursor substrates, respectively [5], [6].

This research team has previously assayed a combination of screening tools from a wide collection of probiotic strains and selecting the best producer of CLA and CLNA isomers - Bifidobacterium breve DSM 20091 - to proceed afterwards with studies on CLA/CLNA-enriched milk. Seeking to explore industrial viability, commercial edible vegetables oils were applied as precursor substrate sources instead, being previously hydrolysed with lipases to enhance the amounts of free LA/ $\alpha$ LNA. After a series of optimization assays, the flaxseed oil (FSO), which is rich in $\alpha$-LNA, provided the highest yield of microbial conjugated FA $(\sim 1 \mathrm{mg} / \mathrm{g})$ at $2 \mathrm{mg} / \mathrm{mL} \alpha$-LNA. After elaborating the new functional milk, the next required step is evaluating its compositional stability throughout storage. Therefore, the aim of the present work was to investigate if a microbial CLA/CLNA-enriched fermented milk is stable, in terms of probiotic viable counts, $\mathrm{pH}$ and fatty acid (FA) profile, at conditions mimicking shelf-life.

Pasteurized cow milk was inoculated with B. breve DSM 20091 and $2 \mathrm{mg} / \mathrm{mL}$ of $\alpha$-LNA (from hydrolysed FSO) or not (control) and distributed by $100 \mathrm{~mL}$ containers for each sampling point and in triplicate. The containers were fermented for $22 \mathrm{~h}$ at $37^{\circ} \mathrm{C}$ under anaerobic conditions. After fermentation, three containers from each substrate condition were separated for further analysis (T0d) and the rest was stored at $4{ }^{\circ} \mathrm{C}$, being well sealed with parafilm and protected from light with aluminium foil. Samples were taken from storage each 7 days until the end of the assay (T28d). At each sampling point, it was performed viable cell counting in cys-MRS agar plates and total microbial count in PCA plates, measure of pH and FA analysis content through GC-FID.

According to the obtained results, after 7 days of storage, viable cell counts of B. breve DSM 20091 decreased significantly, especially in the enriched milk (from 8.09 to $4.51 \log _{10}$ ), and kept decreasing, reaching insignificant counts by the end of the assay. Concerning to total microbial count in PCA, insignificant numbers were detected throughout all storage period at both conditions. In terms of $\mathrm{pH}$, it maintained constant overall (Control: 5.05-5.14; Enriched milk: 4.86-4.99). As for FA profile, in the non-esterified fraction, it was detected higher levels of CLA and CLNA isomers in the enriched milk, and consequently of total polyunsaturated FA (PUFA) as well, at T28d, comparing to all other sampling points, with values of $0.17 \mathrm{mg} / \mathrm{g}$ CLA, $1.11 \mathrm{mg} / \mathrm{g}$ CLNA and $2.04 \mathrm{mg} / \mathrm{g}$ PUFA. In the esterified fraction, was observed variations throughout storage in total saturated FA of control $(7.85-9.18 \mathrm{mg} / \mathrm{g})$, and in total monounsaturated FA of both conditions (Control: 2.23$2.81 \mathrm{mg} / \mathrm{g}$; Enriched milk: 2.19-2.66 mg/g).

In conclusion, a microbial CLA/CLNA-enriched fermented milk is not entirely stable during storage, including its CLA/CLNA content, which was in fact increased. 


\section{References}

[1] G. Fuke and J. L. Nornberg, "Systematic evaluation on the effectiveness of conjugated linoleic acid in human health," Crit. Rev. Food Sci. Nutr., vol. 57, no. 1, pp. 1-7, 2017.

[2] A. A. Hennessy, R. P. Ross, G. F. Fitzgerald, and C. Stanton, "Sources and bioactive properties of conjugated dietary fatty acids," Lipids, vol. 51, no. 4, pp. 377-397, 2016.

[3] P. Shokryzadan et al., "Conjugated linoleic acid: a potent fatty acid linked to animal and human health," Crit. Rev. Food Sci. Nutr., vol. 57, no. 13, pp. 2737-2748, 2017.

[4] A. L. Fontes, L. L. Pimentel, C. D. Simões, A. M. Gomes, and L. M. Rodríguez-Alcalá, "Evidences and perspectives in the utilization of CLNA isomers as bioactive compounds in foods," Crit. Rev. Food Sci. Nutr., vol. 57, no. 12, pp. 26112622, 2017.

[5] B. Yang et al., "Bacterial conjugated linoleic acid production and their applications," Prog. Lipid Res., vol. 68, pp. 2636, 2017.

[6] A. L. Fontes, L. Pimentel, L. M. Rodríguez-Alcalá, and A. Gomes, "Effect of Pufa Substrates on Fatty Acid Profile of Bifidobacterium breve Ncimb 702258 and CLA/CLNA Production in Commercial Semi-Skimmed Milk," Sci. Rep., vol. 8, no. 1, p. 15591, 2018. 